\title{
Pingouin: statistics in Python
}

\author{
Raphael Vallat ${ }^{1}$ \\ 1 Department of Psychology, University of California, Berkeley.
}

DOI: $10.21105 /$ joss.01026

\section{Software}

- Review ¿

- Repository ๘

- Archive ${ }^{\top}$

Submitted: 05 October 2018

Published: 19 November 2018

\section{License}

Authors of papers retain copyright and release the work under a Creative Commons Attribution 4.0 International License (CC-BY).

\section{Summary}

Python is currently the fastest growing programming language in the world, thanks to its ease-of-use, fast learning curve and its numerous high quality packages for data science and machine-learning. Surprisingly however, Python is far behind the R programming language when it comes to general statistics and for this reason many scientists still rely heavily on $\mathrm{R}$ to perform their statistical analyses.

In this paper, we present Pingouin, an open-source Python package aimed at partially filling this gap by providing easy-to-use functions for computing some of the main statistical tests that scientists use on an every day basis. This includes basics functions such as ANOVAs, ANCOVAs, post-hoc tests, non-parametric tests, effect sizes, as well as more advanced functions such as Bayesian T-tests (Rouder, Speckman, Sun, Morey, \& Iverson, 2009), repeated measures correlations (Bakdash \& Marusich, 2017), robust correlations (Pernet, Wilcox, \& Rousselet, 2012) and circular statistics (Berens, 2009), to cite but a few. Pingouin is written in Python 3 and is mostly built on top of the Pandas (McKinney, 2010) library, therefore allowing a fluid integration within a data analysis pipeline. Pingouin comes with an extensive documentation and API as well as with several Jupyter notebook examples.

\section{References}

Bakdash, J. Z., \& Marusich, L. R. (2017). Repeated measures correlation. Front. Psychol., 8, 456.

Berens, P. (2009). CircStat: A MATLAB toolbox for circular statistics. J. Stat. Softw., $31(10), 1-21$.

McKinney, W. (2010). Data structures for statistical computing in python. In S. van der Walt \& J. Millman (Eds.), Proceedings of the 9th python in science conference (pp. 51-56).

Pernet, C. R., Wilcox, R., \& Rousselet, G. A. (2012). Robust correlation analyses: False positive and power validation using a new open source matlab toolbox. Front. Psychol., 3, 606 .

Rouder, J. N., Speckman, P. L., Sun, D., Morey, R. D., \& Iverson, G. (2009). Bayesian $\mathrm{t}$ tests for accepting and rejecting the null hypothesis. Psychon. Bull. Rev., 16(2), $225-237$. 